\title{
The cost-effectiveness of providing antenatal lifestyle advice for women who are overweight or obese: the LIMIT randomised trial
}

Jodie M Dodd ${ }^{1,2^{*}}$, Sharmina Ahmed ${ }^{3,4}$, Jonathan Karnon ${ }^{5}$, Wendy Umberger ${ }^{3,4}$, Andrea R Deussen ${ }^{1}$, Thach Tran ${ }^{1}$, Rosalie M Grivell ${ }^{1,2}$, Caroline A Crowther ${ }^{1,6}$, Deborah Turnbull', Andrew J McPhee ${ }^{8}$, Gary Wittert ${ }^{9}$, Julie A Owens ${ }^{1}$, Jeffrey S Robinson ${ }^{1}$ and For the LIMIT Randomised Trial Group

\begin{abstract}
Background: Overweight and obesity during pregnancy is common, although robust evidence about the economic implications of providing an antenatal dietary and lifestyle intervention for women who are overweight or obese is lacking.

We conducted a health economic evaluation in parallel with the LIMIT randomised trial. Women with a singleton pregnancy, between $10^{+0}-20^{+0}$ weeks, and $\mathrm{BMI} \geq 25 \mathrm{~kg} / \mathrm{m}^{2}$ were randomised to Lifestyle Advice (a comprehensive antenatal dietary and lifestyle intervention) or Standard Care. The economic evaluation took the perspective of the health care system and its patients, and compared costs encountered from the additional use of resources from time of randomisation until six weeks postpartum. Increments in health outcomes for both the woman and infant were considered in the cost-effectiveness analysis. Mean costs and effects in the treatment groups allocated at randomisation were compared, and incremental cost effectiveness ratios (ICERs) and confidence intervals (95\%) calculated. Bootstrapping was used to confirm the estimated confidence intervals, and to generate acceptability curves representing the probability of the intervention being cost-effective at alternative monetary equivalent values for the outcomes avoiding high infant birth weight, and respiratory distress syndrome. Analyses utilised intention to treat principles.
\end{abstract}

Results: Overall, the increase in mean costs associated with providing the intervention was offset by savings associated with improved immediate neonatal outcomes, rendering the intervention cost neutral (Lifestyle Advice Group $\$ 11261.19 \pm \$ 14573.97$ versus Standard Care Group $\$ 11306.70 \pm \$ 14562.02 ; p=0.094)$. Using a monetary value of $\$ 20,000$ as a threshold value for avoiding an additional infant with birth weight above $4 \mathrm{~kg}$, the probability that the antenatal intervention is cost-effective is 0.85 , which increases to 0.95 when the threshold monetary value increases to $\$ 45,000$.

Conclusions: Providing an antenatal dietary and lifestyle intervention for pregnant women who are overweight or obese is not associated with increased costs or cost savings, but is associated with a high probability of cost effectiveness. Ongoing participant follow-up into childhood is required to determine the medium to long-term impact of the observed, short-term endpoints, to more accurately estimate the value of the intervention on risk of obesity, and associated costs and health outcomes.

Trials registration: Australian and New Zealand Clinical Trials Registry (ACTRN12607000161426).

Keywords: Pregnancy, Overweight and obesity, Health economics evaluation, Cost effectiveness analysis, Randomised trial, dietary and lifestyle intervention

\footnotetext{
* Correspondence: jodie.dodd@adelaide.edu.au

${ }^{1}$ School of Paediatrics and Reproductive Health, and The Robinson Research

Institute, The University of Adelaide, Adelaide, Australia

${ }^{2}$ Department of Perinatal Medicine, Women's and Babies Division, The

Women's and Children's Hospital, North Adelaide, Australia

Full list of author information is available at the end of the article
}

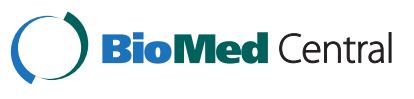

(C) 2015 Dodd et al.; licensee BioMed Central. This is an Open Access article distributed under the terms of the Creative Commons Attribution License (http://creativecommons.org/licenses/by/4.0), which permits unrestricted use, distribution, and reproduction in any medium, provided the original work is properly credited. The Creative Commons Public Domain Dedication waiver (http://creativecommons.org/publicdomain/zero/1.0/) applies to the data made available in this article, unless otherwise stated. 


\section{Background}

It has been estimated that in excess of 1.46 billion adults [1], and 170 million children [2] across the globe are obese. Obesity is a well-recognised contributor to the overall global burden of disease [3], associated with a range of adverse health outcomes including cardiovascular disease, type- 2 diabetes and several cancers. When compared with individuals of normal body mass index, obesity has been conservatively estimated to increase the costs of health care by $30 \%$ for individuals who are obese, compared with those of normal body mass index (BMI) [4]. Furthermore, obesity is estimated to result in more than 36 million disability-adjusted life-years [5], and to contribute directly to in excess of 3.4 million deaths annually [6].

The financial costs of obesity are substantial, an Australian report estimating approximately $\$ 21$ billion annually in 2005 [7], further increasing to more than $\$ 58$ billion per annum in 2008 [8]. Using a broader concept of "cost" to include measures of collective wellbeing, it has been estimated that obesity accounts for $\$ 120$ billion annually, or $8 \%$ of Australia's national annual productivity [9]. Data from the United States indicate $\$ 147$ billion dollars, or $10 \%$ of the country's total health care expenditure was spent on treatment of obesity related complications in 2006 [10], with projections indicating a doubling of costs each decade, representing up to $18 \%$ of total health-care expenditure by 2030 [11].

Obesity represents a significant health burden for women and their infants during pregnancy and childbirth, with almost $50 \%$ of women having a BMI above $25 \mathrm{~kg} / \mathrm{m}^{2}$ on entering pregnancy [12-14]. The effects of high maternal BMI on pregnancy and infant health have been well documented, the risks of adverse outcomes increasing with increasing maternal BMI [15-19]. While the economic implications associated with obesity have been relatively well described in non-pregnant settings $[4,6-8]$, there is more limited information available relating to the healthcare costs associated with obesity during pregnancy. The limited available literature indicates maternal overweight and obesity to be associated with increased cost of healthcare, including prolonged hospitalisation and neonatal nursery care, when compared with women of normal BMI [20-24], estimated to be $23 \%$ higher among women who are overweight, and increasing further to $37 \%$ greater among women who are obese [22].

There is considerable research interest in the evaluation of antenatal interventions provided to women who are overweight or obese to limit gestational weight gain $[25,26]$. However, to our knowledge, there is little robust information available evaluating the economic implications of providing these interventions, either at an individual or population level. The aim of this pre-specified analysis was to evaluate the economic costs and consequences of providing an antenatal lifestyle intervention for women who are overweight or obese, conducted within the context of the LIMIT randomised trial [27].

\section{Methods}

An economic evaluation was conducted in conjunction with the LIMIT randomised trial. The protocol [28] and clinical findings from the LIMIT trial have been published in detail previously $[27,29,30]$. Briefly, eligible women with a singleton pregnancy and BMI $\geq 25 \mathrm{~kg} / \mathrm{m}^{2}$, who were between $10^{+0}$ and $20^{+0}$ weeks' gestation, were recruited from the three major metropolitan maternity hospitals within Adelaide, South Australia. All women provided written informed consent, following approval from the ethics committee at each collaborating hospital including Women's and Children's Health Network HREC, Human Research Ethics Committee (TQEH/ $\mathrm{LMH} / \mathrm{MH}$ ) and Southern Adelaide Clinical HREC. At the time of their first antenatal visit, all women had their height and weight measured, and BMI calculated. Women were then allocated a study number and randomised to receive either 'Lifestyle Advice' or 'Standard Care'.

Women randomised to receive Lifestyle Advice participated in a comprehensive dietary and lifestyle intervention over the course of their pregnancy, which included a combination of dietary, exercise and behavioral strategies, delivered by a research dietician and trained research assistants, as described previously [27]. Within two weeks of randomisation, women attended a planning session with a research dietician. The information presented was subsequently reinforced during contact with the research dietician at 28 weeks' gestation, and with trained research assistants (via telephone call at 22, 24 , and 32 weeks' gestation and a face-face visit at 36 weeks' gestation).

Women randomised to receive Standard Care continued their pregnancy care according to local hospital guidelines, which did not include routine provision of dietary, lifestyle and behavioural advice related to diet or gestational weight gain.

The economic evaluation took the perspective of the health care system, and compared the costs directly associated with the additional use of resources from the time of randomisation until six weeks postpartum. Health outcomes for both the woman and her infant were considered in the clinical trial, but only those outcomes for which the intervention achieved a statistically significant improvement are included in the costeffectiveness analysis, namely high infant birth weight and respiratory distress syndrome. Costs of outpatient occasions of service were calculated as the number of occasions each service was encountered, multiplied by the unit cost for that service. Table 1 presents the unit 


$\begin{aligned} & \text { Table } 1 \text { Unit costs of providing an antenatal lifestyle } \\
& \text { intervention for women who are overweight/obese }\end{aligned}$
\begin{tabular}{ll} 
Item & $\begin{array}{l}\text { Unit Cost } \\
\text { (Australian dollars) }\end{array}$ \\
\hline Antenatal Clinic Visit (Doctor or Midwife) & $\$ 217$ \\
Antenatal General Practitioner Visit $^{+}$ & $\$ 70.30$ \\
Antenatal Anaesthetic Visit $^{*}$ & $\$ 184$ \\
Antenatal Physician Visit $^{*}$ & $\$ 217$ \\
Diabetes Educator Visit $^{*}$ & $\$ 217$ \\
Provision Lifestyle Intervention $^{*}$ & $\$ 195$
\end{tabular}

Service encounters collected from the trial data for all women randomised. Unit costs were derived from the hospital cost databases $\left(^{*}\right)$ and the Medicare Benefits Schedule (+). Patient-level costings were available for all inpatient episodes.

costs for the non-inpatient service encounters for which data were collected for all women randomised, including general practitioner or obstetrician/physician visits, midwifery visits, antenatal anaesthetist consultation, dietician visits, and antenatal emergency department attendance. Unit costs for these items were taken from hospital cost databases and the Medicare Benefits Schedule. Patientlevel inpatient costs for the woman (including delivery), and neonatal care for the infant were available for each woman randomised to the trial utilising computerised inpatient cost information systems.

Mean costs and effectives in the treatment groups allocated at the time of randomisation were compared, and incremental cost effectiveness ratios (ICERs) and confidence intervals (95\%) calculated. Bootstrapping (using 1,000 resamples) was used to confirm the estimated confidence intervals, and to generate acceptability curves that represent the probability of the intervention being costeffective at alternative monetary equivalent values for the main outcomes (avoiding high infant birth weight, and avoiding infant respiratory distress syndrome) [31].

Health related quality of life of the woman was assessed at baseline and at regular intervals to 4 months postpartum, by self-completion of the SF36-Health Survey Questionnaire [32]. The 36 items were combined into eight multi-item summary scores, and the Bayesian nonparametric conversion algorithm was used to generate health-related quality of life (utility) weights on a 0 (dead) to 1 (full health) scale [33]. Differences in utility profiles over the trial follow-up period were compared between the intervention and control group.

Consistent with the primary analysis of the LIMIT trial [27], economic analyses were adjusted for the stratification variables BMI category $\left(25.0-29.9 \mathrm{~kg} / \mathrm{m}^{2}\right.$ vs $\left.\geq 30.0 \mathrm{~kg} / \mathrm{m}^{2}\right)$, parity (parity 0 vs parity 1 or more), and centre of recruitment, and additionally for maternal age, and socioeconomic status. All data were analysed according to the original treatment assignment using intention to treat principles.

\section{Results}

Of the 2,212 women randomised to the LIMIT trial, 1,108 were randomised to the Lifestyle Advice Group, and 1,104 to the Standard Care Group. There were no statistically significant differences in the baseline characteristics of women randomised between the two treatment groups (Table 2) [27], or in health service encounters, with the exception of visits to the diabetes educator which were increased in women receiving Lifestyle Advice (Table 3). For clinical infant outcomes, as reported previously, infants born to women following lifestyle advice were significantly less likely to have birth weight above $4.0 \mathrm{~kg}$ (Lifestyle Advice 164/1075 (15.22\%) versus Standard Care 201/1067 (18.79\%); aRR 0.82; 95\% CI 0.68 to 0.99 ; Number Needed to Treat (NNT) 28; 95\% CI 15 to 263; $\mathrm{p}=0.04)$ [27]. Furthermore, infants born to women following lifestyle advice were significantly less likely to have birth weight above $4.5 \mathrm{~kg}(2 \cdot 15 \%$ versus $3 \cdot 69 \%$; aRR 0.59 ; $95 \%$ CI 0.36 to $0.98 ; \mathrm{p}=0.04$ ), or respiratory distress syndrome $(1 \cdot 22 \%$ versus $2 \cdot 57 \%$; aRR 0 . $47 ; 95 \% \mathrm{CI} 0 \cdot 24$ to $0 \cdot 90 ; \mathrm{p}=0 \cdot 02$ ), particularly moderate or severe disease, and had a shorter length of postnatal hospital stay $(3.94 \pm 7.26$ days versus $4.41 \pm$ 9.87 days; adjusted ratio of means 0.89 ; $95 \%$ CI $0.82-0.97$; $\mathrm{p}=0.006)$ when compared with infants born to women who received standard care [30]. No significant differences in the health-related quality of life (utility) of women were identified between the two treatment groups over the follow-up period.

\section{Health service use and direct outpatient- and inpatient costs for study participants}

The mean cost associated with providing the Lifestyle Advice was $\$ 320.12 \pm \$ 130.97$. Mean antenatal outpatient costs were $\$ 83.40$ higher for women randomised to the Lifestyle Advice Group, compared with women receiving Standard Care (Lifestyle Advice Group \$2116.39 \pm $\$ 895.14$ versus Standard Care $\$ 2032.99 \pm \$ 805.63$; $\mathrm{p}=0.022$ ) (Table 4). However, mean costs were lower, albeit not reaching statistical significance, for women in the Lifestyle Advice Group, reflecting a mean of \$281.01 savings in inpatient costs (Lifestyle Advice Group $\$ 6125.04 \pm \$ 4640.78$ versus Standard Care Group $\$ 6404.05 \pm \$ 5274.54 ; \mathrm{p}=0.185)$, and $\$ 168.02$ savings in infant hospitalisation costs (Lifestyle Advice Group $\$ 2699.64 \pm \$ 13068.48$ versus Standard Care Group $\$ 2867.66 \pm \$ 12344.64 ; \mathrm{p}=0.756$ ).

Overall, therefore, the increase in mean costs associated with providing the intervention was offset by savings associated with improved immediate neonatal outcomes, rendering the intervention cost neutral (Lifestyle Advice Group \$11261.19 $\pm \$ 14573.97$ versus Standard Care Group \$11306.70 \pm \$14562.02; $\mathrm{p}=0.094)$. 
Table 2 Baseline characteristics at trial entry [27]

\begin{tabular}{|c|c|c|c|}
\hline Characteristic & $\begin{array}{l}\text { Lifestyle advice } \\
\left(\mathrm{N}=1105^{* *}\right)\end{array}$ & $\begin{array}{l}\text { Standard care } \\
\left(N=1097^{* *}\right)\end{array}$ & $\begin{array}{l}\text { Total } \\
\left(N=2202^{* *}\right)\end{array}$ \\
\hline Maternal Age (Years) $^{*}$ & $29.3(5.4)$ & $29.6(5.6)$ & $29.4(5.5)$ \\
\hline Gestational Age at Entry (Weeks) ${ }^{+}$ & $14.0(11.9-17.0)$ & $14.1(11.9-17.0)$ & $14.1(11.9-17.0)$ \\
\hline Body Mass Index $\left(\mathrm{kg} / \mathrm{m}^{2}\right)^{+}$ & $31.0(28.1-35.9)$ & $31.1(27.7-35.6)$ & $31.1(27.9-35.8)$ \\
\hline \multicolumn{4}{|l|}{ Body Mass Index Category" } \\
\hline BMI 25.0-29.9 & $458(41.4)$ & $468(42.7)$ & $926(42.1)$ \\
\hline BMI 30.0-34.9 & $326(29.5)$ & $318(29.0)$ & $644(29.2)$ \\
\hline BMI 35.0-39.9 & $202(18.3)$ & $183(16.7)$ & $385(17.5)$ \\
\hline $\mathrm{BMI}>=40.0$ & $119(10.8)$ & $128(11.7)$ & $247(11.2)$ \\
\hline Public Patient ${ }^{\#}$ & $1081(97.8)$ & $1067(97.3)$ & $2148(97.5)$ \\
\hline Weight $(\mathrm{kg})^{*}$ & $88.6(17.3)$ & $88 \cdot 2(17.6)$ & $88.4(17.4)$ \\
\hline Height $(\mathrm{cm})^{*}$ & $164.9(6.6)$ & $164 \cdot 8(6.5)$ & $164.8(6.6)$ \\
\hline \multicolumn{4}{|l|}{ Race $^{\#}$} \\
\hline Caucasian & $995(90.0)$ & $998(91.0)$ & $1993(90.5)$ \\
\hline Asian & $26(2.4)$ & $34(3.1)$ & $60(2.7)$ \\
\hline Indian & $40(3.6)$ & $35(3.2)$ & $75(3.4)$ \\
\hline Other & $44(4.0)$ & $30(2.7)$ & $74(3.4)$ \\
\hline Smoker ${ }^{\#}$ & $154(13.9)$ & $126(11.5)$ & $280(12.7)$ \\
\hline Nulliparous" ${ }^{\#}$ & $457(41.4)$ & $441(40.2)$ & $898(40.8)$ \\
\hline Previous Preterm Birth ${ }^{\#}$ & $57(5.2)$ & $59(5.4)$ & $116(5.3)$ \\
\hline Previous Pre-eclampsia" & $46(4.2)$ & $51(4.6)$ & $97(4.4)$ \\
\hline Previous Stillbirth" & $13(1.2)$ & $6(0.5)$ & $19(0.9)$ \\
\hline Previous Neonatal Death ${ }^{\#}$ & $11(1.0)$ & $7(0.6)$ & $18(0.8)$ \\
\hline Previous Caesarean Section ${ }^{\#}$ & $197(17.8)$ & $214(19.5)$ & $411(18.7)$ \\
\hline Family History of Diabetes" & $288(26.1)$ & $290(26.4)$ & $578(26.2)$ \\
\hline Family History of Hypertension ${ }^{\#}$ & $389(35.2)$ & $369(33.6)$ & $758(34.4)$ \\
\hline Family History of Heart Disease ${ }^{\#}$ & $187(16.9)$ & $179(16.3)$ & $366(16.6)$ \\
\hline \multicolumn{4}{|l|}{ Index of Socio-economic Disadvantage^ } \\
\hline Unknown & $2(0.2)$ & $1(0.1)$ & $3(0.1)$ \\
\hline Quintile 1 (Most Disadvantaged) & $340(30.8)$ & $321(29.3)$ & $661(30.0)$ \\
\hline Quintile 2 & $271(24.5)$ & $264(24.1)$ & $535(24.3)$ \\
\hline Quintile 3 & $173(15.7)$ & $174(15.9)$ & $347(15.8)$ \\
\hline Quintile 4 & $150(13.6)$ & $178(16.2)$ & $328(14.9)$ \\
\hline Quintile 5 (Least Disadvantaged) & $169(15.3)$ & $159(14.5)$ & $328(14.9)$ \\
\hline
\end{tabular}

* = mean and standard deviation.

${ }^{+}=$median and interquartile range.

\# = number and \%.

$\wedge=$ Socioeconomic index as measured by SEIFA.

** = Includes all women randomised who did not withdraw consent to use their data.

\section{Costs and consequences, and cost effectiveness of providing lifestyle advice}

The unadjusted bootstrapped estimate of the difference in the proportion of infants with birth weight above $4 \mathrm{~kg}$ was 0.019 (95\% CI 0.011 to 0.025$)$, and infant respiratory distress syndrome was 0.0123 (95\% CI 0.009 to 0.017 ). The incremental cost effectiveness ratio in preventing one additional infant with birth weight above $4 \mathrm{~kg}$ was $\$ 2395.26$ (95\% CI $\$ 2198$ to $\$ 2411$ ), and in preventing one additional infant with moderate to severe respiratory distress syndrome was $\$ 3700$ (95\% CI $\$ 3589$ to $\$ 3755$ ).

Figures 1 and 2 represent the probability that providing an antenatal lifestyle intervention is cost-effective, in preventing both birth weight above $4 \mathrm{~kg}$ and moderate 
Table 3 Health services utilisation from randomisation to 6 weeks post-partum

\begin{tabular}{|c|c|c|c|c|}
\hline \multirow[t]{2}{*}{ Service } & \multirow{2}{*}{$\begin{array}{l}\text { Lifestyle advice } \\
\mathrm{N}=1,105\end{array}$} & \multirow{2}{*}{$\begin{array}{l}\text { Standard care } \\
\mathrm{N}=1,097\end{array}$} & \multirow{2}{*}{$\begin{array}{l}\text { Adjusted treatment } \\
\text { effect }(95 \% \mathrm{Cl})\end{array}$} & \multirow{2}{*}{$\begin{array}{l}\text { Adjusted } \\
\mathrm{P} \text { value }\end{array}$} \\
\hline & & & & \\
\hline \multicolumn{5}{|l|}{ Antenatal Services } \\
\hline Antenatal Clinic Visit* & $7.47( \pm 2.68)$ & $7.38( \pm 2.65)$ & $0.09(-0.15$ to 0.31$)$ & 0.46 \\
\hline General Practitioner Visit* & $1.09( \pm 2.66)$ & $1.12( \pm 2.74)$ & $-0.05(-0.28$ to 0.18$)$ & 0.67 \\
\hline Antenatal Anaesthetic Visit* & $0.21( \pm 0.45)$ & $0.22( \pm 0.45)$ & $-0.01(-0.49$ to 0.02$)$ & 0.49 \\
\hline Antenatal Physician Visit* & $0.65( \pm 1.52)$ & $0.57( \pm 1.44)$ & $-0.08(-0.05$ to 0.20$)$ & 0.24 \\
\hline Diabetes Educator Visit* & $0.38( \pm 1.40)$ & $0.25( \pm 0.99)$ & $-0.14(-0.24$ to 0.03$)$ & 0.01 \\
\hline \multicolumn{5}{|l|}{ Birthing Services } \\
\hline Induction of Labour ${ }^{+}$ & $390(36.25)$ & 378 (35.39) & $1.03(0.92$ to 1.15$)$ & 0.63 \\
\hline Caesarean birth $^{+}$ & $370(34.46)$ & $389(36.50)$ & 0.95 (0.85 to 1.06$)$ & 0.34 \\
\hline \multicolumn{5}{|l|}{ Neonatal Services } \\
\hline Admission to neonatal nursery ${ }^{+}$ & $394(36.61)$ & $385(36.05 \%)$ & $1.00(0.90$ to 1.12$)$ & 0.99 \\
\hline Need for respiratory support ${ }^{+}$ & 65 (6.09) & 77 (7.20\%) & 0.84 (0.61 to 1.15$)$ & 0.27 \\
\hline
\end{tabular}

* = Data presented as mean and standard deviation.

$+=$ Data presented as number and percentage.

Treatment effect represents adjusted difference in means (or relative risk ratio) with $95 \%$ confidence intervals.

Adjusted analyses included the stratification variables BMI category, parity and centre, in addition to adjustment for maternal age, and socioeconomic status.

to severe respiratory distress syndrome, given alternative equivalent monetary values.

If a value of $\$ 20,000$ is used as a threshold value for avoiding an additional infant with birth weight above $4 \mathrm{~kg}$, the probability of the intervention being cost-effective is 0.85 , compared with 0.15 in providing standard care (i.e. 1 minus the probability of cost-effectiveness for the intervention). This probability of intervention cost-effectiveness reaches almost 0.95 for the Lifestyle Advice Group if the maximum threshold willingness to pay increases from $\$ 20,000$ to $\$ 45,000$. Using the same threshold value of $\$ 20,000$ for avoiding an additional infant with moderate or severe respiratory distress syndrome, the probability of cost-effectiveness is 0.64 in providing lifestyle advice. Additionally if we consider the maximum threshold value as $\$ 45,000$, the probability of cost-effectiveness increases from 0.64 to nearly 0.73 for the Lifestyle Advice Group.

\section{Discussion}

Our randomised trial is the largest reported to date evaluating the effect of an antenatal lifestyle intervention for women who are overweight or obese during pregnancy, and the first to report a robust economic evaluation of the associated costs and consequences. The findings of this

Table 4 Cost of health services utilisation after randomisation for women and their infants

\begin{tabular}{|c|c|c|c|c|}
\hline Service component & $\begin{array}{l}\text { Lifestyle advice } \\
\mathrm{N}=1,105\end{array}$ & $\begin{array}{l}\text { Standard care } \\
\mathrm{N}=1,097\end{array}$ & $\begin{array}{l}\text { Adjusted treatment effect } \\
(95 \% \mathrm{Cl})\end{array}$ & $\begin{array}{l}\text { Adjusted } \\
P \text { value }\end{array}$ \\
\hline \multicolumn{5}{|l|}{ Maternal Outpatient Services } \\
\hline Antenatal Clinic Visit & $\$ 1580.47(\$ 626.50)$ & $\$ 1558.16(\$ 625.02)$ & $\$ 22.30(-\$ 27.05$ to $\$ 65.39)$ & 0.416 \\
\hline \multicolumn{5}{|l|}{ (Obstetrician/Physician/Midwife) } \\
\hline General Practitioner Visit & $\$ 118.97(\$ 204.49)$ & $\$ 115.42(\$ 210.07)$ & $\$ 3.55(-\$ 14.33$ to $\$ 16.59)$ & 0.689 \\
\hline Antenatal Anaesthetic Visit & $\$ 38.41(\$ 83.16)$ & $\$ 40.83(\$ 82.48)$ & $-\$ 2.43(-\$ 8.58$ to $\$ 3.25)$ & 0.477 \\
\hline Diabetes Educator Visit & $\$ 74.42(\$ 289.80)$ & $\$ 47.47(\$ 203.46)$ & $\$ 26.95(\$ 7.24$ to $\$ 49.11)$ & 0.008 \\
\hline Emergency Department Presentation & $\$ 305.06(\$ 466.84)$ & $\$ 272.18(\$ 380.69)$ & $\$ 32.88(-\$ 6.92$ to $\$ 55.68)$ & 0.127 \\
\hline Total Outpatient Services & $\$ 2116.39(\$ 895.14)$ & $\$ 2032.99(\$ 805.63)$ & $\$ 83.40(\$ 38.25$ to $\$ 140.50)$ & 0.022 \\
\hline Provision of Antenatal Dietary \& Lifestyle Advice & $\$ 320.12(\$ 130.97)$ & N/A & $\mathrm{N} / \mathrm{A}$ & N/A \\
\hline Maternal Inpatient Services & $\$ 6125.04(\$ 4640.78)$ & $\$ 6406.05(\$ 5274.54)$ & $-\$ 281.01(-\$ 289.31$ to $\$ 448.38)$ & 0.185 \\
\hline Neonatal Inpatient Services & $\$ 2699.64$ (\$13068.48) & $\$ 2867.66(\$ 12344.64)$ & $-\$ 168.02(-\$ 748.22$ to $\$ 18.47)$ & 0.756 \\
\hline Total Costs Incurred & $\$ 11261.19$ (\$14573.97) & $\$ 11306.70(\$ 14562.02)$ & $-\$ 45.51(-\$ 1349.26$ to $\$ 1003.54)$ & 0.094 \\
\hline
\end{tabular}

Data presented as mean and standard deviation.

Treatment effect represents adjusted difference in means with $95 \%$ confidence intervals.

Adjusted analyses included the stratification variables BMI category, parity and centre, in addition to adjustment for maternal age, and socioeconomic status. 


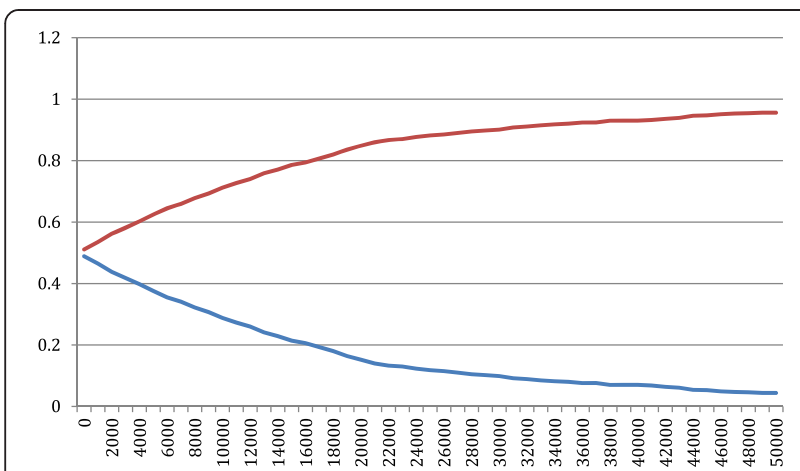

Figure 1 Cost-effectiveness acceptability curve for infant birth weight below $\mathbf{4} \mathbf{~ k g}$. Legend: Red line represents the cost effectiveness acceptability of achieving infant birth weight below $4 \mathrm{~kg}$ in the Standard Care Group; Blue line represents the cost effectiveness acceptability of achieving infant birth weight below $4 \mathrm{~kg}$ in the Lifestyle Advice Group.

health economic analysis conducted in parallel with a large-scale randomised trial indicate that the provision of an antenatal dietary and lifestyle intervention for pregnant women who are overweight or obese is not associated with statistically significantly increased costs or cost savings. While providing access to the intervention was associated with an increase in antenatal and dietician costs, this was offset by the savings associated with improved immediate infant birth outcomes and reduced hospitalisation costs. Using a monetary value of $\$ 20,000$ as a threshold value for avoiding an additional infant with birth weight above $4 \mathrm{~kg}$, the probability of cost-effectiveness of providing the antenatal intervention was 0.85 , increasing to 0.95 with a monetary value of $\$ 45,000$.

While the economic implications associated with overweight and obesity among non-pregnant individuals has been relatively well described [4,6-8], there is more

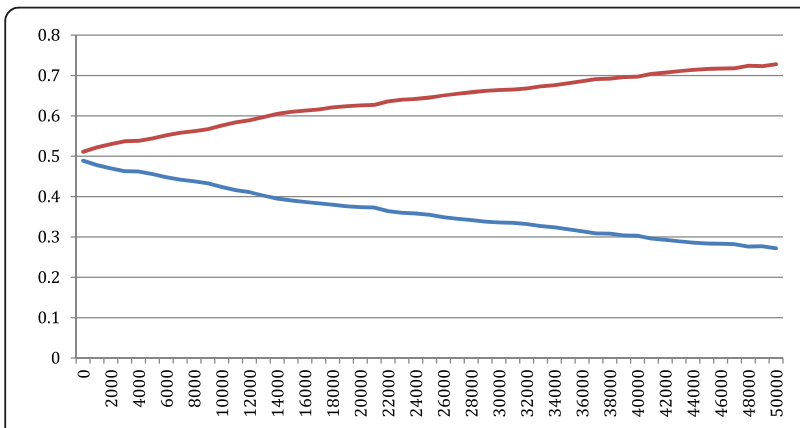

Figure 2 Cost-effectiveness acceptability curve for preventing one infant developing moderate to severe respiratory distress syndrome. Legend: Red line represents the cost effectiveness acceptability of preventing one infant developing moderate to severe respiratory distress syndrome in the Standard Care Group; Blue line represents the cost effectiveness acceptability of preventing one infant developing moderate to severe respiratory distress syndrome in the Lifestyle Advice Group. limited information available relating to the health care costs associated with obesity during pregnancy. However, available literature indicates maternal overweight and obesity to be associated with increased costs of providing antenatal care, increased number of admissions and length of hospitalisation, and an overall increase in health care cost, when compared with women of normal BMI [20-24], reflecting in part an increase in the risk of obesity related maternal and infant complications [20]. Specifically, the mean total costs associated with pregnancy and postpartum care have been estimated to be $23 \%$ higher among women who are overweight, increasing to $37 \%$ higher among women who are obese, when compared with women of normal BMI [22], with suggestions that interventions costing less than $£ 1171$ per person could be cost effective in reducing healthcare utilisation costs among pregnant women who are obese [22]. The cost of providing dietary and lifestyle advice as described in the LIMIT randomised trial was approximately $\$ 320$, or $£ 179$ per woman randomised, well below the above threshold.

The equally important and potentially more significant medium to longer-term implications of providing such a cost-neutral and effective antenatal intervention lies in the well-described association between high infant birth weight and subsequent risk of both childhood [34,35] and adulthood overweight and obesity [36,37], derived from several population-based cohort studies. Population cohorts have also identified consequent associations between high infant birth weight and subsequent cardiometabolic risk factors, including higher blood pressure, among children [38] which may persist into early adulthood [39]. Observational data from the United States involving 7,738 adolescents [40] highlights a significantly higher prevalence of obesity among children with birth weight above $4 \mathrm{~kg}$. While children of high birth weight represented $12 \%$ of the cohort, this was disproportionately increased among children who were obese at age 14 years, where $36 \%$ of individuals had birth weight over $4 \mathrm{~kg}[40]$.

The economic implications associated with childhood obesity are considerable [41], with data from Australia identifying medical costs within the first five years of schooling to be $\$ 9.8$ million greater for overweight or obese children at age 5 years, when compared with children of normal BMI [42]. Economic data from the United States indicate an increase in use of prescription medication, and both emergency and outpatient presentations among children who are overweight or obese, reflecting a cost of $\$ 14.1$ billion annually [43], increasing by another $\$ 238$ million per annum when accounting for increased inpatient admissions [44]. Conservative estimates suggest medical costs alone which are directly attributable to high childhood BMI are approximately 
$\$ 6.24$ billion, with the loss of more than 2 million quality adjusted life years [41].

The medium to longer-term consequences of obesity in adulthood are substantial, with obesity considered the sixth most important factor contributing the global burden of disease [3], accounting for approximately 3.4 million adult deaths annually [6], through an increased risk of hypertension, cardiovascular disease, type- 2 diabetes and malignancy [3]. The economic implications of treating complications related to adult obesity have variably been estimated at more than $\$ 58$ billion, with $\$ 35.6$ billion reflecting indirect costs [45]. Projections from the US highlight an increase in the proportion of total health-care expenditure to treat obesity and its complications [11], although as highlighted by others, direct international comparisons are exceedingly difficult [46].

There are limitations to our study. As reported previously [27], our trial population was predominantly of white Caucasian background and of high social disadvantage. Furthermore, $60 \%$ of eligible women declined to participate, reflecting both a lack of interest and time due to other commitments. The demographic characteristics of the women who participated in the LIMIT trial are similar to those of the broader population of women giving birth in South Australia [47], and therefore provide reassurance that our findings have wider clinical applicability. However, the transferability of the findings of our economic evaluation will likely vary with the similarities to both our trial population and health care system. While we used a standard assessment of social disadvantage in the SEIFA index, we were not able to assess in more detail the effects of occupation and household income. Furthermore, the analysis took the perspective of the health care institution, limiting our ability to evaluate household opportunity costs and financial implications from a societal perspective.

\section{Conclusions}

Clearly, prevention, rather than treatment of obesity and obesity related complications should be the goal. Increasingly, there is recognition that the intra-uterine environment has a key role in the development of later health and disease [48], and therefore represents a critical period in the subsequent programming of obesity. Any antenatal intervention which successfully reduce the risk of high infant birth weight as was demonstrated in the LIMIT randomised trial [27], and particularly those which are cost effective as reported here, represent a public health strategy of significant potential in tackling the increasing problem of overweight and obesity, both in the short and longer-term [49,50]. Ongoing follow-up of participants into childhood will therefore be of great importance to determine the longer-term impact of the intervention on risk of obesity, and the associated economic implications.

\section{Competing interests}

The authors declare that they have no competing interests.

\section{Authors' contributions}

JMD, SA, JK, WU, ARD, T, RMG, CAC, DT, AJM, GW, JAO, and JSR are all members of the LIMIT randomised trial group. The primary investigator of the LIMIT randomised trial (JMD) prepared the initial draft of the manuscript, had full access to all of the study data, and takes responsibility for the integrity of the data, and the accuracy of the data analysis. JMD, SA, JK and WU were responsible for conducting the statistical analyses. All members of the LIMIT randomised trial group listed above were involved in the study concept and design of the trial, supervision of conduct of the trial, the acquisition of data, the analysis and interpretation of data, critical revision of the manuscript for important intellectual content, and provides approval of the final submitted version.

\section{Authors' information}

Jodie M Dodd and Sharmina Ahmed are equal first authorship.

\section{Acknowledgements}

This project was funded by a four-year project grant from the National Health and Medical Research Council (NHMRC), Australia (ID 519240).

JM Dodd is supported through a NHMRC Practitioner Fellowship (ID 627005). RM Grivell is supported through a NHMRC Early Career Fellowship (ID 1073514). Infrastructure support was provided by The University of Adelaide, and the Women's and Children's Hospital, Flinders Medical Centre, and Lyell McEwin Hospital, Adelaide.

The funders had no role in the study design, data collection and analysis, decision to publish, or preparation of the manuscript.

The following persons and institutions (except where indicated, in Adelaide, South Australia) participated in the LIMIT Trial:

Steering Group -JM Dodd (Chair), D Turnbull, A McPhee, RM Grivell, C Crowther, M Gillman (Obesity Prevention Program, and Harvard University, Boston, Massachusetts, USA), G Wittert, JA Owens, JS Robinson Co-ordinating Team -JM Dodd, A Deussen, RM Grivell, L Yelland, L Moran, C Cramp, A Newman, L Kannieappian, S Hendrijanto, M Kelsey, J Beaumont, C Danz, J Koch, A Webber, C Holst, K Robinson, S Zhang, V Ball, K Ball, H Deussen, N Salehi, R Bartley, R Stafford-Green, S Ophel, M Cooney, M Szmeja, A Short, A Melrose, S Han, I Mohamad, L Chapple

Statistical Analyses - L Yelland

Serious Adverse Events Committee - RM Grivell, J Svigos, V Bhatia, N Manton Writing Group -JM Dodd, D Turnbull, A McPhee, A Deussen, RM Grivell, L Yelland, C Crowther, G Wittert, JA Owens, JS Robinson

Collaborating Hospitals (total number of women recruited from each site in parentheses), *indicates named associate investigator for the NHMRC grant. - Flinders Medical Centre (South Australia) (669): J McGavigan*, R Bryce, S Coppi, C Fanning, G Hannah, M Ignacio, H Pollard, F Schmidt, Y Shinners - Lyell McEwin Hospital (South Australia) (505): G Dekker*, S Kennedy-Andrews, R Beaven, J Niven, S Burgen, J Dalton, N Dewhurst, L Forst, V Mugg, C Will, H Stone, G Wedlock

- Women's and Children's Hospital (South Australia) (1,038): JM Dodd, JS Robinson, A Deussen, C Crowther*, C Wilkinson*, H Purcell, J Wood, D Press, K Ralph, S Donleavy, S Seager, F Gately, A Jolly, L Lahnstein, S Harding, K Daw, M Hedges, R Fraser-Trumble, G Wedlock

We are indebted to the 2,212 women who participated in this randomised trial.

\section{Author details}

${ }^{1}$ School of Paediatrics and Reproductive Health, and The Robinson Research Institute, The University of Adelaide, Adelaide, Australia. ${ }^{2}$ Department of Perinatal Medicine, Women's and Babies Division, The Women's and Children's Hospital, North Adelaide, Australia. ${ }^{3}$ Agricultural and Food Economics, Global Food Studies, Faculty of the Professions, The University of Adelaide, Adelaide, Australia. ${ }^{4}$ Women's and Children's Health Research Institute, North Adelaide, Australia. ${ }^{5}$ School of Population Health, The University of Adelaide, Adelaide, Australia. ${ }^{6}$ Liggins Institute, University of Auckland, Auckland, New Zealand. ${ }^{7}$ School of Psychology, The University of Adelaide, Adelaide, Australia. ${ }^{8}$ Department of Neonatal Medicine, Women's and Babies Division, The Women's and Children's Hospital, North Adelaide, Australia. ${ }^{9}$ School of Medicine, The University of Adelaide, Adelaide, Australia. 
Received: 22 September 2014 Accepted: 23 February 2015 Published online: 11 March 2015

\section{References}

1. Finucane MM, Stevens GA, Cowan MJ, Danaei G, Lin JK, Paciorek CJ, et al. National, regional, and global trends in body-mass index since 1980: systematic analysis of health examination surveys and epidemiological studies with 960 country-years and 9. 1 million participants. Lancet. 2011;377(9765):557-67.

2. Lobstein T, Baur L, Uauy R, for the International Obesity Task Force. Obesity in children and young people: a crisis in public health. Obes Rev. 2004;5 Suppl 1:4-104.

3. Ezzati M, Lopez AD, Rodgers A, Vander Hoorn S, Murray CJ, for the Comparative Risk Assessment Collaborating Group. Selected major risk factors and global and regional burden of disease. Lancet. 2002;360:1347-60.

4. Withrow D, Alter DA. The economic burden of obesity worldwide: a systematic review of the direct costs of obesity. Obes Rev. 2011;12(2):131-41.

5. World Health Organisation. Global health risks: mortality and burden of disease attributable to selected major risks. Geneva: World Health Organisation WHO; 2009.

6. World Health Organisation: Obesity and overweight Fact Sheet. http://www.who. int/mediacentre/factsheets/fs311/en/ 2014

7. Access Economics. The economic costs of obesity. Diabetes Australia. 2006

8. Access Economics. The growing cost of obesity in 2008: three years on. Diabetes Australia. 2008.

9. The Herald/Age - Lateral Economics Index of Australia's Wellbeing. http://lateraleconomics.com.au/wp-content/uploads/2014/02/Fairfax-LateralEconomics-Index-of-Australias-Wellbeing-Final-Report.pdf.

10. Roehr B. "Soda tax" could help tackle obesity, says US director of public health. BMJ 2009, 339:b3176. (http://www.bmj.com/content/339/bmj.b3176 (Published 04 August 2009)).

11. Wang Y, Beydoun MA, Liang L, Caballero B, Kumanyika SK. Will all Americans become overweight or obese? Estimating the progression and cost of the US obesity epidemic. Obesity. 2008;16(10):2323-30.

12. National Institute for Health and Clinical Excellence: Weight management before, during and after pregnancy [http://www.nice.org.uk/guidance/ph27/ chapter/2-public-health-need-and-practice].

13. Scheil W, Scott J, Catcheside B, Sage L. Pregnancy outcome in South Australia 2010. Pregnancy Outcome Unit, SA Health. Adelaide: Government of South Australia; 2012.

14. IOM (Institute of Medicine) and NRC (National Research Council): Weight Gain During Pregnancy: Reexamining the Guidelines. Washington, DC: The National Academies Press; 2009. [http://www.nap.edu/catalog/12584/ weight-gain-during-pregnancy-reexamining-the-guidelines]

15. Callaway LK, Prins JB, Chang AM, Mclntyre HD. The prevalence and impact of overweight and obesity in an Australian obstetric population. MJA. 2006;184(2):56-9.

16. Dodd JM, Grivell RM, Nguyen A-M, Chan A, Robinson JS. Maternal and perinatal health outcomes by body mass index category. ANZJOG. 2011;51(2):136-40.

17. Abenhaim HA, Kinch RA, Morin L, Benjamin A, Usher R. Effect of prepregnancy body mass index categories on obstetrical and neonata outcomes. Arch Gynecol Obstet. 2007;275:39-43.

18. Cedergren MI. Maternal morbid obesity and the risk of adverse pregnancy outcome. Obstet Gynecol. 2004;103:219-24.

19. Cnattingius S, Bergstrom R, Lipworth L, Kramer MS. Prepregnancy weight and the risk of adverse pregnancy outcomes. New Engl J Med. 1998;338:147-52.

20. Denison FC, Norwood P, Bhattacharya S, Duffy A, Mahmood T, Morris C, et al. Association between maternal body mass index during pregnancy, short-term morbidity, and increased health service costs: a populationbased study. BJOG. 2014;121(1):72-81.

21. Galtier-Dereure F, Boegner C, Bringer J. Obesity and pregnancy: complications and cost. Am J Clin Nutr. 2000;71:S1242-8.

22. Morgan KL, Rahman MA, Macey S, Atkinson MD, Hill RA, Khanom A, et al Obesity in pregnancy: a retrospective prevalence-based study on health service utilisation and costs on the NHS. BMJ Open. 2014;4(2):e003983. doi:10.1136/bmjopen-2013-003983.

23. Trasande L, Lee M, Liu Y, Weitzman M, Savitz D. Incremental charges, costs, and length of stay associated with obesity as a secondary diagnosis among pregnant women. Med Care. 2009;47(10):1046-52.
24. Heslehurst N, Simpson H, Ells LJ, Rankin J, Wilkinson J, Lang R, et al. The impact of maternal BMI status on pregnancy outcomes with immediate short-term obstetric resource implications: a meta-analysis. Obes Rev. 2008;9(6):635-83.

25. Dodd JM, Grivell RM, Crowther CA, Robinson JS. Antenatal interventions for overweight or obese pregnant women: a systematic review of randomised trials. Br J Obstet Gynaecol. 2010;117(11):1316-26.

26. Oteng-Ntim E, Varma R, Croker H, Poston L, Doyle P. Lifestyle interventions for overweight and obese pregnant women to improve pregnancy outcome: systematic review and meta-analysis. BMC Med. 2012;10:47.

27. Dodd JM, Turnbull DA, MCPhee AJ, Deussen AR, Grivell RM, Yelland LN, et al. Antenatal lifestyle advice for women who are overweight or obese: the LIMIT randomised trial. BMJ. 2014;348:g1285.

28. Dodd JM, Turnbull DA, McPhee AJ, Wittert G, Crowther CA, Robinson JS. Limiting weight gain in overweight and obese women during pregnancy to improve health outcomes: the LIMIT randomised controlled trial. BMC Pregnancy Childbirth. 2011;11:79.

29. Dodd JM, Cramp CS, Sui Z, Yelland LN, Deussen AR, Grivell RM, et al. Effects of antenatal lifestyle advice for women who are overweight or obese on maternal diet and physical activity: the LIMIT randomised trial. BMC Med. 2014;12:161. http://www.biomedcentral.com/1741-7015/12/161.

30. Dodd JM, McPhee AJ, Turnbull DA, Yelland LN, Deussen AR, Grivell RM, et al. The effect of antenatal lifestyle advice for women who are overweight or obese on neonatal health: the LIMIT randomised trial. BMC Med. 2014;12:163. http://www.biomedcentral.com/1741-7015/12/163.

31. Glick H, Briggs A, Polsky D. Quantifying stochastic uncertainty and presenting results of cost effectiveness analyses. Expert Rev Pharmacoecon Outcomes Res. 2001;1:25-36.

32. Ware JE, Sherbourne CD. The MOS 36 item short form health survey (SF36) conceptual framework and item selection. Med Care. 1992;30:473-83.

33. Kharroubi SA, Brazier JE, Roberts J, O'Hagan A. Modelling SF-6D health state preference data using a nonparametric Bayesian method. J Health Econ. 2007;26(3):597-612.

34. Kitsantas P, Gaffney KF. Risk profiles for overweight/obesity among preschoolers. Early Hum Dev. 2010;86:563-8.

35. Rooney BL, Mathiason MA, Schauberger CW. Predictors of obesity in childhood, adolescence, and adulthood in a birth cohort. Maternal Child Health J. 2010, DOl10.1007/s10995-010-0689-1.

36. Schellong K, Schulz S, Harder T, Plagemann A. Birth weight and long term overweight risk: systematic review and meta-analysis including 643,902 persons from 66 studies and 26 countries globally. PLoS One. 2012;7(10):e47776. doi:47710.41371/journal.pone.0047776.

37. Yu ZB, Han SP, Zhu GZ, Zhu C, Wang XJ, Cao XG, et al. Birth weight and subsequent risk of obesity: a systematic review and meta-analysis. Obes Rev. 2011;12(7):525-42.

38. Fraser A, Tilling K, Macdonald-Wallis C, Sattar N, Brion MJ, Benfield L, et al. Association of maternal weight gain in pregnancy with offspring obesity and metabolic and vascular traits in childhood. Circulation. 2010;121:2557-64.

39. Mamun AA, Kinarivala M, O'Callaghan MJ, Williams GM, Najman JM, Callaway LK. Associations of excess weight gain during pregnancy with long-term maternal overweight and obesity: evidence from 21y postpartum follow-up. Am J Clin Nutr. 2010;91:1336-41.

40. Cunningham SA, Kramer MR, Narayan KM. Incidence of childhood obesity in the United States. N Engl J Med. 2014;370(5):403-11.

41. Trasande L. How much should we invest in preventing childhood obesity? Health Aff. 2010;29(3):372-8.

42. Au N. The Health Care Cost Implications of Overweight and Obesity during Childhood. Health Serv Res 2011, Sep 23. doi:10.1111/j.1475-6773.2011.01326.x. [Epub ahead of print].

43. Trasande $L$, Chatterjee $S$. The impact of obesity on health service utilization and costs in childhood. Obesity. 2009;17(9):1749-54.

44. Trasande L, Liu Y, Fryer G, Weitzman M. Effects of childhood obesity on hospital care and costs, 1999-2005. Health Aff. 2009;28(4):4.

45. Colagiuri S, Lee CM, Colagiuri R, Magliano D, Shaw JE, Zimmet PZ, et al. The cost of overweight and obesity in Australia. Med J Aust. 2010;192(5):260-4.

46. Dee A, Kearns K, O'Neill C, Sharp L, Staines A, O'Dwyer V, et al. The direct and indirect costs of both overweight and obesity: a systematic review. BMC Res Notes. 2014;7:242.

47. Scheil W, Scott J, Catcheside B, Sage L, Kennare R. Pregnancy Outcome in South Australia 2012. SA Health Pregnancy Outcome Unit South Australia. Adelaide: Government of South Australia; 2014. 
48. Barker DJP. Mothers, babies and disease in later life. 2nd ed. London: Harcourt Brace \& Co; 1998.

49. Catalano PM. Obesity and pregnancy-the propagation of a viscous cycle? J Clin Endocrinol Metab. 2003;88(8):3505-6.

50. Poston L. Healthy eating in pregnancy - always a good idea, now with more supporting evidence. BMJ. 2014;348:g1739.

Submit your next manuscript to BioMed Central and take full advantage of:

- Convenient online submission

- Thorough peer review

- No space constraints or color figure charges

- Immediate publication on acceptance

- Inclusion in PubMed, CAS, Scopus and Google Scholar

- Research which is freely available for redistribution 